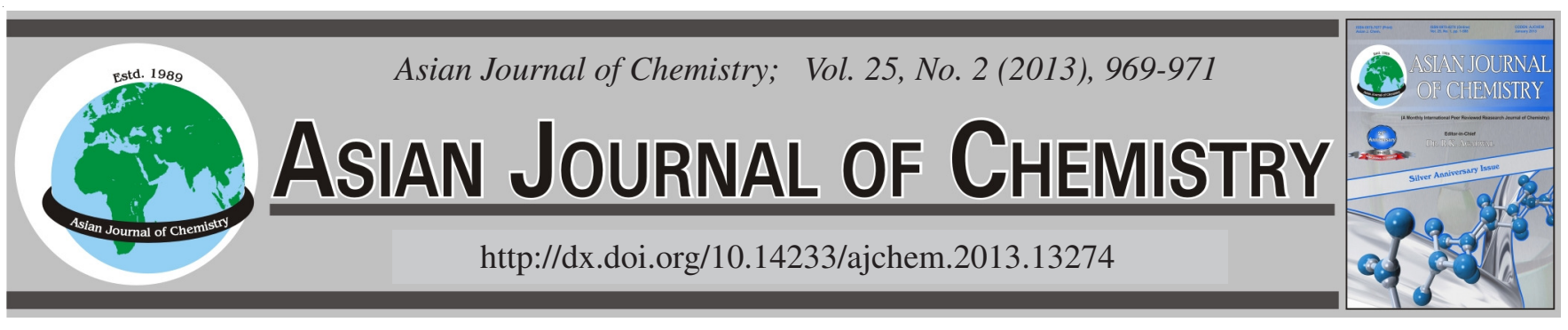

\title{
Growth and Characterization of Bis(thiourea) Cadmium Acetate Single Crystal: An Efficient Semiorganic Non-linear Optical Single Crystal
}

\author{
Johanan Christian Prasana ${ }^{1}$, A. Anuradha ${ }^{2}$, J. Madhavan ${ }^{3, *}$ and P. Sagayaraj ${ }^{3}$
}

\author{
${ }^{1}$ Department of Physics, Madras Christian College, Chennai-600 059, India \\ ${ }^{2}$ Department of Physics, Queen Mary's College, Chennai-600 004, India \\ ${ }^{3}$ Department of Physics, Loyola College, Chennai-600 034, India \\ *Corresponding author: E-mail: jmadhavang@yahoo.com
}

(Received: 14 December 2011;

Accepted: 27 August 2012)

AJC-12023

\begin{abstract}
Bis(thiourea) cadmium acetate, a metal semiorganic non-linear optical crystal, was synthesized from thiourea and cadmium acetate using double distilled water. The synthesized salt was used to grow bulk size crystals. The XRD analysis confirmed that bis(thiourea)cadmium acetate has orthorhombic crystal structure. The UV transmission study indicates that there is a very low absorption in the visible light region. FTIR analysis suggests the presence of functional groups in the grown crystal. Thermal studies reveal that the grown crystal has considerable thermal stability and it can be exploited for industrial applications. The non-linear optical study confirms frequency doubling of the sample using Nd:YAG laser.
\end{abstract}

Key Words: Bis(thiourea) cadmium acetate, Single crystal, Growth, Non-linear optical crystal.

\section{INTRODUCTION}

In the last decade, organic nonlinear optical crystals with aromatic rings have attracted much attention because of their high nonlinearity, fast response and tailor made flexibility ${ }^{1}$ However, the shortcomings of aromatic crystals, such as poor physicochemical stability, low hardness and cleavage tendency hinder their device applications.

In order to keep the merits and overcome the shortcomings of organic materials, a class of non-linear optical crystals namely, organometallic or semiorganic crystals have been developed ${ }^{2}$. Ideally the large non-linearities of $\pi$-conjugated organics and the favourable crystal growth characteristics and mechanical properties of ionic salts can be combined into a single non-linear optical crystal ${ }^{3}$. Extended $\pi$-conjugated networks in organic systems with the largest nonlinearities invariably have significant absorption in the visible region of the spectrum ${ }^{4}$. Consequently, for SHG into the blue-near-UV regions, more transparent and less extensively delocalized organics must be considered (e.g., urea) at the expense of decreasing the nonlinearity. The thiourea molecule is an interesting inorganic matrix modifier due to its large dipole moment and its ability to form an extensive network of hydrogen bonds. Bis(thiourea) cadmium acetate has been identified as an organometallic nonlinear optical crystal, which is a new semiorganic non-linear optical material synthesized by combining thiourea (which is typical polar molecule) with cadmium acetate ${ }^{5}$.

\section{EXPERIMENTAL}

Synthesis and growth: Bis(thiourea) cadmium acetate (BTCA) salt was synthesized by dissolving thiourea and cadmium acetate taken in the molar ratio $2: 1$ in double distilled water. The reaction formula is:

$$
\begin{aligned}
\mathrm{Cd}\left(\mathrm{CH}_{3} \mathrm{COO}\right)_{2}+2\left[\mathrm{CS}\left(\mathrm{NH}_{2}\right)_{2}\right] \longrightarrow \\
\mathrm{Cd}\left[\mathrm{CS}\left(\mathrm{NH}_{2}\right)_{2}\right]_{2} \cdot\left(\mathrm{CH}_{3} \mathrm{COO}\right)_{2}
\end{aligned}
$$

Solubility of bis(thiourea) cadmium acetate in double distilled water was determined at different temperatures (30, $35,40,45$ and $50^{\circ} \mathrm{C}$ ) with continuous stirring. After attaining the saturation, the equilibrium concentration of the solute was estimated gravimetrically. The solubility curve of bis(thiourea)cadmium acetate is shown in Fig. 1.

The saturated solution of bis(thiourea) cadmium acetate was carefully prepared using double distilled water based on the solubility data. The solution was continuously stirred for 2 to 3 days in order to maintain uniform concentration gradient in the crystallizer. After stirring the solution, it was filtered and transferred to a $250 \mathrm{~mL}$ beaker covered with perforated plastic thin sheet. The solution was allowed to evaporate at $305 \mathrm{~K}$.

Due to spontaneous nucleation seed crystals were seen within the period of 7 to 10 days. Transparent good quality seed crystals with perfect shape and free from macro defects were taken and immersed in the mother solution using nylon 


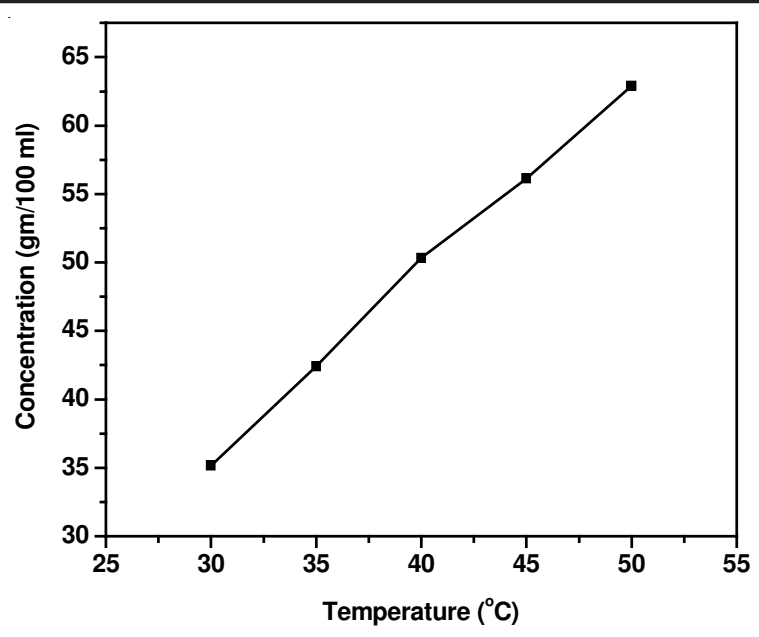

Fig. 1. Solubility curve for bis(thiourea) cadmium acetate

thread. Single crystal of size upto $19 \mathrm{~mm} \times 8 \mathrm{~mm} \times 7 \mathrm{~mm}$ was harvested in a period of 25 to 30 days and Fig. 2 shows the photograph of the as grown crystal of bis(thiourea) cadmium acetate. The crystal is highly transparent and free from visible inclusions.

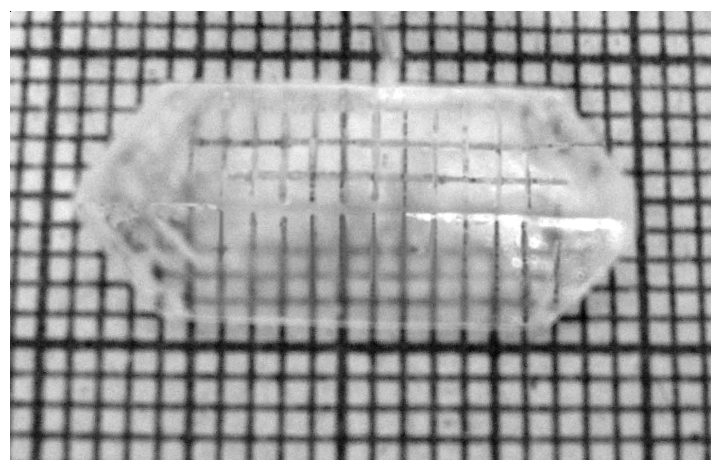

Fig. 2. Photograph of bis(thiourea) cadmium acetate single crystal

Single crystal X-ray diffraction analysis: $B i$ (thiourea) cadmium acetate crystal structure was studied using single crystal X-ray diffraction analysis. The bis(thiourea) cadmium acetate crystal falls under orthorhombic crystal structure and values of the cell parameters are presented in Table-1. The observed data agrees well with the earlier work ${ }^{5}$.

TABLE-1

CRYSTAL DATA OF BIS(THIOUREA) CADMIUM ACETATE SINGLE CRYSTAL

\begin{tabular}{ll}
\hline Crystal formula & $\mathrm{Cd}\left[\mathrm{CS}\left(\mathrm{NH}_{2}\right)_{2}\right]_{2} \cdot\left(\mathrm{CH}_{3} \mathrm{COO}\right)_{2}$ \\
Crystal system & Orthorhombic \\
$\mathrm{a}(\AA)$ & $7.5854(3)$ \\
$\mathrm{b}(\AA)$ & $11.8195(4)$ \\
$\mathrm{c}(\AA)$ & $15.5043(3)$ \\
$\mathrm{V}(\AA)^{3}$ & $1390.05(4)$ \\
$\alpha=\beta=\gamma$ & $90^{\circ}$ \\
\hline
\end{tabular}

\section{RESULTS AND DISCUSSION}

The FTIR spectral analysis of bis(thiourea) cadmium acetate reveals the effect of coordination on vibrational bands of the ligand in the metal complex. Fig. 3 shows the 4000-400 $\mathrm{cm}^{-1}$ region in the FT-IR spectrum of bis(thiourea) cadmium acetate. In the high wavenumber region $300-3400 \mathrm{~cm}^{-1}$, there are several peaks due to $\mathrm{N}-\mathrm{H}$ stretching. A comparison of bis(thiourea) cadmium acetate wavenumbers with thiourea is presented in Table-2. The peaks at 1495 and $1110 \mathrm{~cm}^{-1}$ are due to $\mathrm{CN}$ asymmetric and symmetric stretching vibration, respectively. The peak at $1412 \mathrm{~cm}^{-1}$ is attributed CS asymmetric stretching vibration. The CS symmetric stretching vibration is observed at $725 \mathrm{~cm}^{-1}$. The other modes of vibrations of bis(thiourea) cadmium acetate are assigned and presented in the same table. It is evident from the spectrum of bis(thiourea) cadmium acetate, that the $\mathrm{CN}$ stretching vibrations of thiourea are shifted to higher frequencies whereas, the CS stretching vibrations of thiourea are shifted to lower frequencies. Hence, thiourea has coordinated to the cadmium through sulphur in bis(thiourea) cadmium acetate.

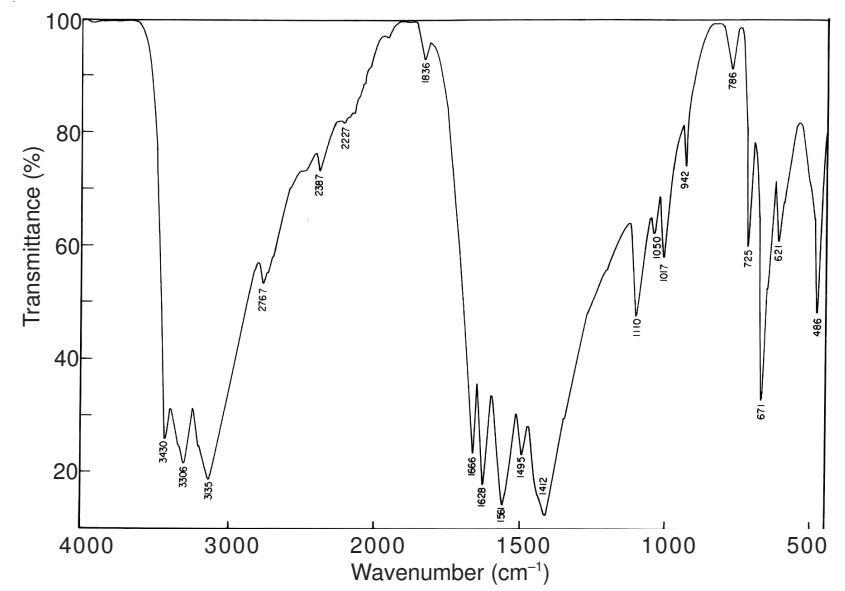

Fig. 3. FTIR spectrum of bis(thiourea) cadmium acetate

TABLE-2

WAVENUMBER ASSIGNMENT OF BIS(THIOUREA) CADMIUM ACETATE (BTCA) WITH THIOUREA

\begin{tabular}{|c|c|c|}
\hline \multicolumn{2}{|c|}{ Wavenumber $\left(\mathrm{cm}^{-1}\right)$} & \multirow{2}{*}{ Assignment } \\
\hline Thiourea & BTCA & \\
\hline $3380,3279,3090$ & $3430,3306,3135$ & N-H Stretching vibrations \\
\hline 1627 & 1666 & $\mathrm{NH}_{2}$ Asymmetric stretching \\
\hline 1472 & 1495 & $\mathrm{CN}$ - Asymmetric stretching \\
\hline 1417 & 1495 & CS- Asymmetric stretching \\
\hline 1089 & 1110 & CN-Symmetric stretching \\
\hline 740 & 725 & CS- Symmetric stretching \\
\hline 640 & 621 & NCS- Asymmetric bending \\
\hline 494 & 486 & NCS- symmetric bending \\
\hline
\end{tabular}

Optical transmission studies: The optical transmission range and the transparency cut-off are important for NLO materials. The optical transmission spectrum of bis(thiourea) cadmium acetate was recorded using a HITACHI U-2010 spectrophotometer in the range 200-1100 nm and is shown in Fig. 4. It is evident from the spectrum that bis(thiourea) cadmium acetate has high percentage of transmission in the entire visible region. The UV transparency cut-off wavelength of grown crystals occurs below $290 \mathrm{~nm}$, which is expected to provide a better range of applications.

NLO test: For the SHG efficiency measurements, microcrystalline material of KDP was used for comparison Kurtz et al. ${ }^{6}$. When a laser input of $6.2 \mathrm{~mJ}$ was passed through bis(thiourea) cadmium acetate, second harmonic signal of $532 \mathrm{~nm}$ 


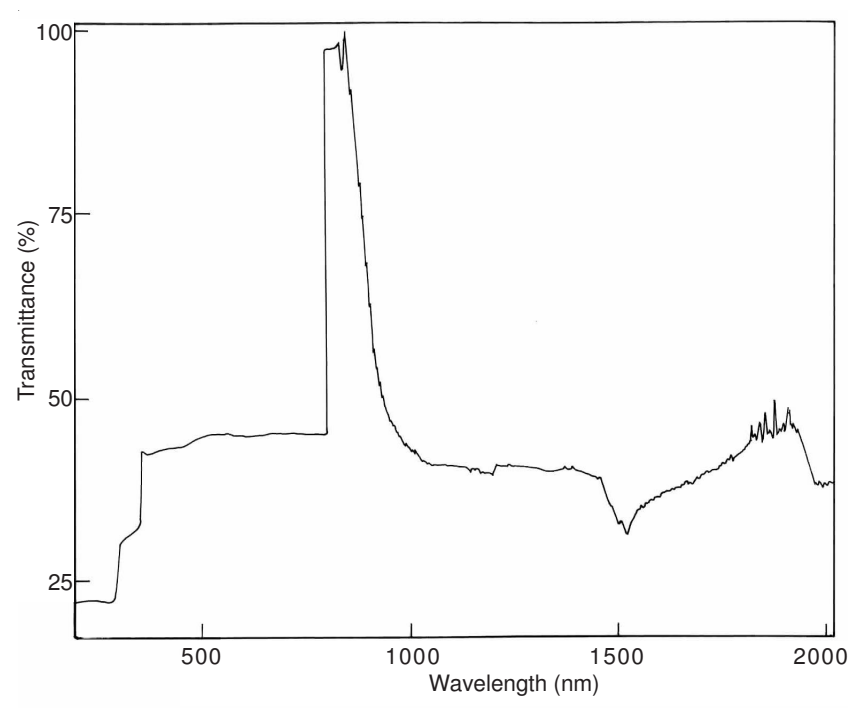

Fig. 4. Optical transmission spectrum of bis(thiourea) cadmium acetate

is produced. The second harmonic signal of $192 \mathrm{mV}$ was obtained for bis(thiourea) cadmium acetate with reference to KDP $(275 \mathrm{mV})$. Thus the SHG efficiency of bis(thiourea) cadmium acetate crystal is nearly 0.7 times than KDP.

Thermal studies: The TG-DTG traces of bis(thiourea) cadmium acetate were recorded between 20 to $1000{ }^{\circ} \mathrm{C}$ at a heating rate of $20 \mathrm{~K} / \mathrm{min}$. using SDT Q600 thermal analyzer. The experiment was performed in nitrogen atmosphere. The resulting thermograms are shown in Fig. 5, which suggest

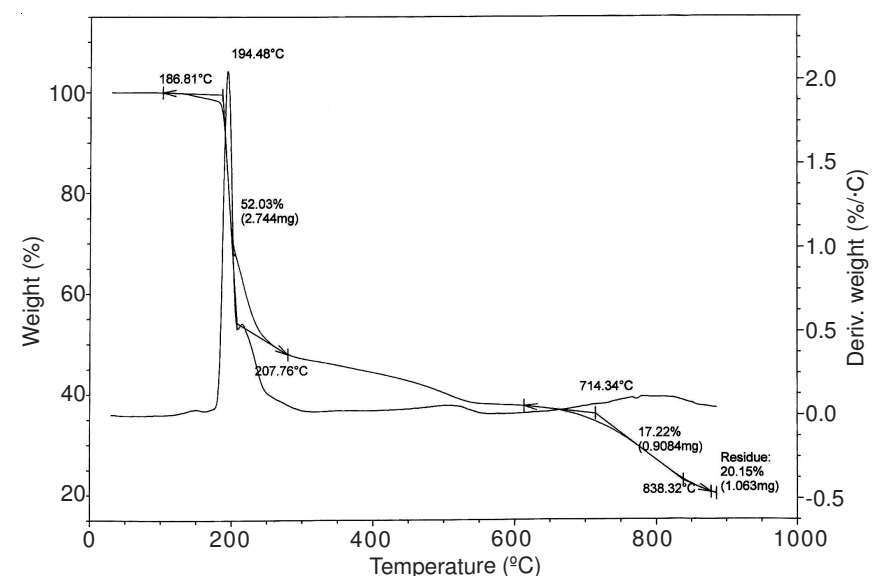

Fig. 5. TG-DTG traces of bis(thiourea) cadmium acetate single crystal nearly 2 stages of decomposition. The maximum decomposition temperature occurs at $194.48^{\circ} \mathrm{C}$, which may be due to the removal of hydrogen from the thiourea molecules. During the first and second stages of decomposition, the weight losses are found to be 52.03 and $17.22 \%$, respectively. The final residue is found to be $20.15 \%$ at $886^{\circ} \mathrm{C}$. Hence, the grown crystal is thermally stable up to $194.48^{\circ} \mathrm{C}$.

\section{Conclusion}

Single crystals of bis(thiourea) cadmium acetate were grown from aqueous solution at room temperature. Solubility of bis(thiourea) cadmium acetate was investigated for double distilled water. Bulk crystals were grown by slow evaporation method. Crystal of size $19 \mathrm{~mm} \times 8 \mathrm{~mm} \times 7 \mathrm{~mm}$ is grown with reasonable growth rate. The structure of bis(thiourea) cadmium acetate was confirmed by single crystal XRD. FTIR studies ascertain the coordination of sulphur with metals. The transmission spectrum of this crystal shows a high transmission in the visible region. Second harmonic generation of bis(thiourea) cadmium acetate was confirmed by Kurtz powder technique. Thermal studies reveal that the grown crystal undergoes two stages of decomposition.

\section{REFERENCES}

1. J. Madhavan, S. Aruna, A. Anuradha, D.P. Anand, I.V. Potheher, K. Thamizharasan and P. Sagayaraj, Opt. Mater., 29, 1211 (2007).

2 J. Madhavan, S. Aruna, K. Ambujam, A.J.A. Pragasam, S.M. Ravikumar, M.G. Mohamed and P. Sagayaraj, Cryst. Res. Tech., 41, 997 (2006).

3 D. Sankar, P.P. Kumar and J. Madhavan, Physica B, 405, 1233 (2010).

4 P.P. Kumar, V. Manivannan, P. Sagayaraj and J. Madhavan, Bull. Mater. Sci., 32, 431 (2009).

5 N.P. Rajesh, V. Kannan, M. Ashok, K. Sivaji, P.S. Raghavan and P. Ramasamy, J. Cryst. Growth, 262, 561 (2004).

$6 \quad$ S.K. Kurtz and T.T. Perry, J. Appl. Phys., 39, 3798 (1968). 